TECHNICAL NOTE
I. Saatci
S. Geyik
K. Yavuz
S. Cekirge

\section{X-Configured Stent-Assisted Coiling in the Endovascular Treatment of Complex Anterior Communicating Artery Aneurysms: A Novel Reconstructive Technique}

\begin{abstract}
SUMMARY: Incomplete initial occlusion rates and relatively less long-term durability remain as major problems of endovascular treatment of cerebral aneurysms. Stent-assisted coiling is a well-established current solution to overcome these problems. Double-stent treatment with a Y-configuration has been previously reported to improve the application of coiling to wide-neck bifurcation aneurysms. Herein, a novel technique of $\mathrm{X}$-configured stent-assisted coiling for treatment of wide-neck and complex AcomA aneurysms is described.
\end{abstract}

ABBREVIATIONS: AcomA = anterior communicating artery; $\mathrm{ACT}=$ activated clotting time; $\mathrm{CTA}=$ CT angiography; ICA = internal carotid artery
L arge series have addressed the impact of the neck size and complex anatomic features of aneurysms on 2 major drawbacks of endovascular coiling, defined as "incomplete initial occlusion rate" and "relatively less long-term durability." The use of self-expanding stents designed specifically for cerebral artery applications can assist in the endovascular treatment of complex wide-neck aneurysms and in achieving more durable long-term results. ${ }^{3,4}$

AcomA aneurysms have a significant importance due to their higher incidence and complex anatomic features, often associated with a wide neck and variety of anomalies. ${ }^{5}$

In this technical note, we describe $\mathrm{X}$-configured stentassisted coiling for treatment of wide-neck and complex AcomA aneurysms, for which otherwise there would be no endovascular treatment option.

\section{Materials and Methods}

Five patients harboring wide-neck complex AcomA aneurysms were treated by using the $\mathrm{X}$-configured stent-assisted coil-embolization technique. The patient population consisted of 3 women and 2 men. Patients' mean age was 44.6 years, with a range between 35 and 58 years. All of the aneurysms were unruptured, and none of them had mass effect. Three aneurysms were small $(<10 \mathrm{~mm})$, and the remaining 2 were large $(\geq 10 \mathrm{~mm})$. In all patients, X-configured stentassisted coiling was the initial treatment strategy, with no patients having any prior treatment attempt. Concurrent with the application of the technique, there was no associated hypoplasia/aplasia of the A1 segment in any of the patients. Because each aneurysm not only incorporated the junction of A1 and A2 bilaterally but also involved the AcomA itself, both balloon remodeling and single-stent placement techniques were deemed unsatisfactory.

All procedures were performed with the patient under general anesthesia with biplane flat-panel state-of-the-art angiographic systems having 3D and intraoperative angiographic CT scanners (Artis zee; Siemens, Erlangen, Germany). Bilateral femoral access was ob-

Received December 23, 2009; accepted after revision February 22, 2010.

From Hacettepe University, Department of Radiology, Ankara, Turkey.

Please address correspondence to Serdar Geyik, MD, Hacettepe University, Department of Radiology, Ankara 06100, Turkey; e-mail: drserdarg@hotmail.com

DOI 10.3174/ajnr.A2111 tained in all patients, and 90-cm introducer sheaths (Arrow International, Reading, Pennsylvania) were placed in both common carotid arteries. We advanced 6F guiding catheters (Envoy; Cordis Neurovascular, Miami Lakes, Florida) to the bilateral ICAs subsequently. Then, rotational angiograms and 3D reconstructions were obtained for all patients, demonstrating the complex anatomy of the aneurysms.

We obey a strict antiaggregation and intraprocedural anticoagulation protocol in our patients undergoing stent placement, particularly dual-stent placement. In this protocol, dual antiplatelet therapy with aspirin (300 mg per day) and clopidogrel are used for premedication 1 week before the procedure with a loading dose of 600-mg clopidogrel; $75 \mathrm{mg}$ per day is continued for 6 months after the endovascular therapy. All patients are examined for clopidogrel sensitivity by using the VerifyNow P12Y12 assay (Accumetrics, San Diego, California). The procedure is not performed if the patient does not have a response of $40 \%$ at minimum (ie, $40 \%$ platelet inhibition) to the clopidogrel. If the loading dose and 1 week's use of clopidogrel do not provide this level of platelet inhibition, we reload the patient and have them use $1 \times 150 \mathrm{mg}$ per day and retest the inhibition. If the second test shows that the patient is still a low responder, the antiaggregation medicine is switched to ticlopidine. However, ticlopidine is not used as the primary line of treatment due to its rare but well-known side effect of bone morrow suppression. Following the control angiogram obtained at 6 months, clopidogrel is discontinued and lifelong use of aspirin is prescribed.

In this small series of patients, this described protocol of antiplatelet regimen was strictly applied. Anticoagulation protocol during the procedure consisted of intravenous administration of heparin with close monitoring of blood ACT levels, which were adjusted according the platelet inhibition level, and the ACT level was kept between 2 and 3 times that of the basal level. Heparin was discontinued after the procedure.

After the evaluation of 2D and 3D angiograms, the sequence of stent placement was decided. The side of the first stent was determined according to the angle between the A1-AcomA complex and the contralateral A2; the A2 with a sharper angle was to be stented before the one with wider angle. On the basis of this decision, a Prowler Select Plus (Cordis Neurovascular) microcatheter with a 0.021-inch inner diameter was placed across the aneurysm neck, ex- 

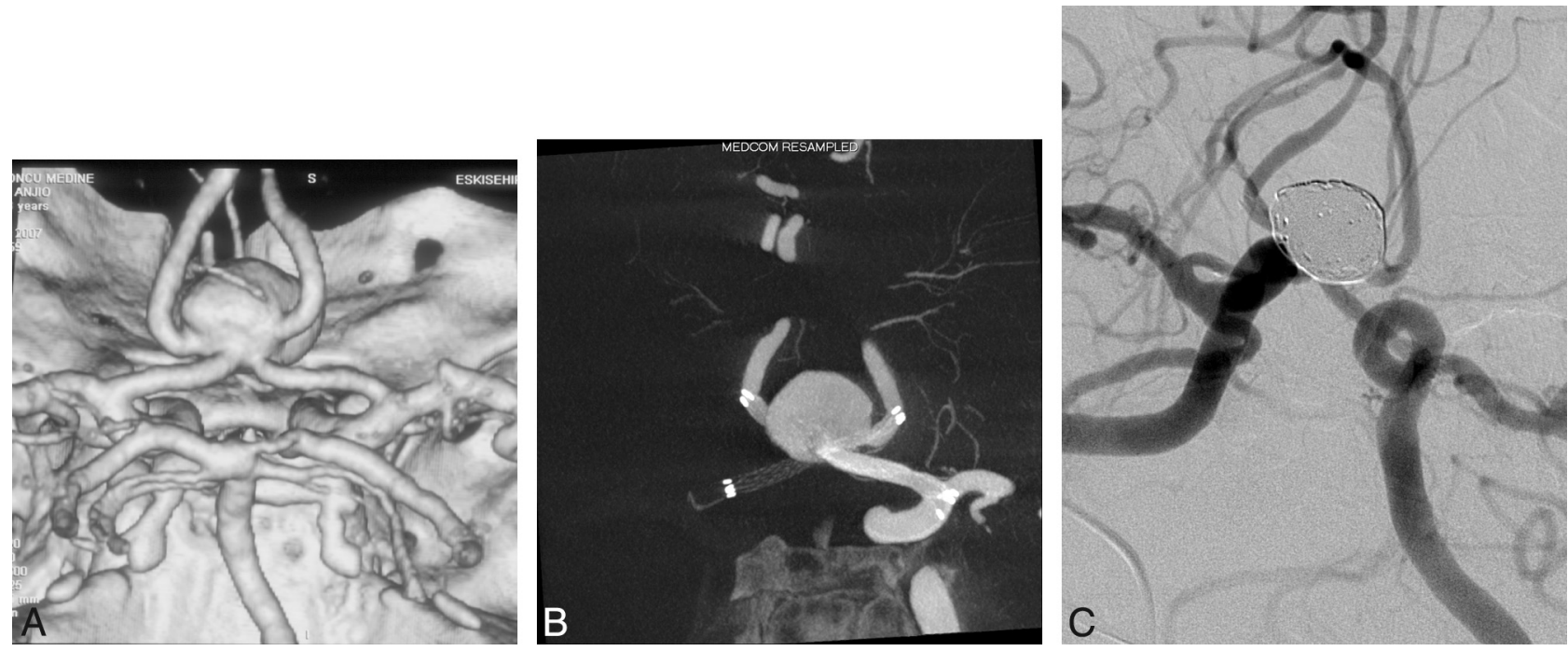

Fig 1. 3D reconstruction image of CTA shows an AcomA aneurysm $(A)$ treated with X-configured stent placement demonstrated in DynaCT angiography (B). C, A 2-year control angiography shows complete occlusion of the aneurysm.

tending from the contralateral A2 to the ipsilateral A1 segment, crossing through the AcomA. Then, an SL-10 microcatheter (Boston Scientific, Fremont, California) or an Echelon 10 microcatheter (ev3, Irvine, California) was jailed into the aneurysm sac, also through the ipsilateral A1, when possible, before the deployment of the first stent. The first stent was then deployed from the contralateral A2 to the ipsilateral A1. The same or a different Prowler Select Plus microcatheter was navigated through the other ICA, and with the help of various guidewires as necessary (0.016 inch and 0.018 inch; Terumo, Tokyo, Japan), it was passed through 1 of the struts of the first stent and placed extending from the contralateral A2 to the ipsilateral A1.

Different combinations of $4.5 \times 22,28$, or $37 \mathrm{~mm}$ Double Enterprise stents (Cordis Neurovascular) were used to create an X-configuration in 4 patients. In the remaining patient, a Solitaire stent of $4 \times$ $20 \mathrm{~mm}$ (ev3) and an Enterprise stent of $4.5 \times 22 \mathrm{~mm}$ were used in combination. The size of the first stent was calculated to allow adequate stent-segment purchase proximal and distal to the A1-A2 junction point to preclude the risk of stent dislodgement during the manipulation of the guidewire for the second stent or navigation of the second stent itself.

The aneurysms were coiled with a variety of bare platinum coils; however, softer coils were preferred to avoid kickback of the microcatheter, which would result in recatheterization of the aneurysm sac. In all patients, we did not densely pack the aneurysms with coils. This decision was intentional on the basis of the confidence provided by our large Y-configuration stent-placement experience offering very durable occlusion rates. The femoral access sites were closed with an Angio-Seal closure device (St. Jude Medical, Minnetonka, Minnesota).

\section{Results}

In all of the patients in this series, dual-stent placement was performed with no technical failure or undesirable event, including artery dissection; failure of deployment, partially or totally, which may obviate the resheathing of the stent; displacement of the first stent while navigating the second through; kinking of the stent, compromising the flow; inability to coil the sac following the stent placement; and so forth. In all patients, we were able to coil the sac to a satisfactory degree through the jailed microcatheter, with no need for recatheterization of the sac, which would have been, then, through the true lumen of the stent passing through the interstices of the overlapping stent segments. There was no procedural or postprocedural neurologic complication in any of the patients. In 1 patient, a significant groin hematoma developed, requiring blood transfusion. All patients underwent 6-month control angiography, and 2 patients had additional 2-year control angiography. All follow-up angiograms revealed complete aneurysm occlusion with no stenosis.

\section{Illustrative Case I}

A 42-year-old woman presented with headache, and her CTA revealed an AcomA aneurysm of $15 \times 12 \mathrm{~mm}$. The $3 \mathrm{D}$ reconstruction image showed that the AcomA aneurysm had a very wide neck involving both A1-A2 junctions with both A2s originating from the aneurysm sac (Fig 1A). Dual stent support was deemed necessary for endosaccular coiling. The patient was premedicated with clopidogrel and aspirin, as described, and $64 \%$ platelet inhibition was obtained. With the patient under general anesthesia, the bilateral ICAs were catheterized with 6 F Envoy guiding catheters (Cordis Neurovascular). 3D angiographic imaging was performed. and working projections were defined. The procedure was performed under the guidance of roadmap images obtained with simultaneous bicarotid contrast injections. After a Prowler Select Plus microcatheter was navigated from the left $\mathrm{A} 1$ to the right $\mathrm{A} 2$, a microcatheter was jailed into the aneurysm sac and an Enterprise stent of $4.5 \times 28 \mathrm{~mm}$ was deployed from the right A2 to left A1. With a 0.016 -inch gold-tip microguidewire (Terumo), the same Prowler microcatheter was navigated from the right A1 to left A2, crossing through the struts of first stent, and placed successfully. A DynaCT (Siemens) angiography was performed to show the satisfactory opening of the X-configured stents (Fig $1 B$ ). Then, the aneurysm sac was coiled with bare platinum detachable coils. The procedure and the postoperative period were uneventful, and the patient was discharged home in the next day. Two-year control angiography (Fig 1C) confirmed complete obliteration of the aneurysm 

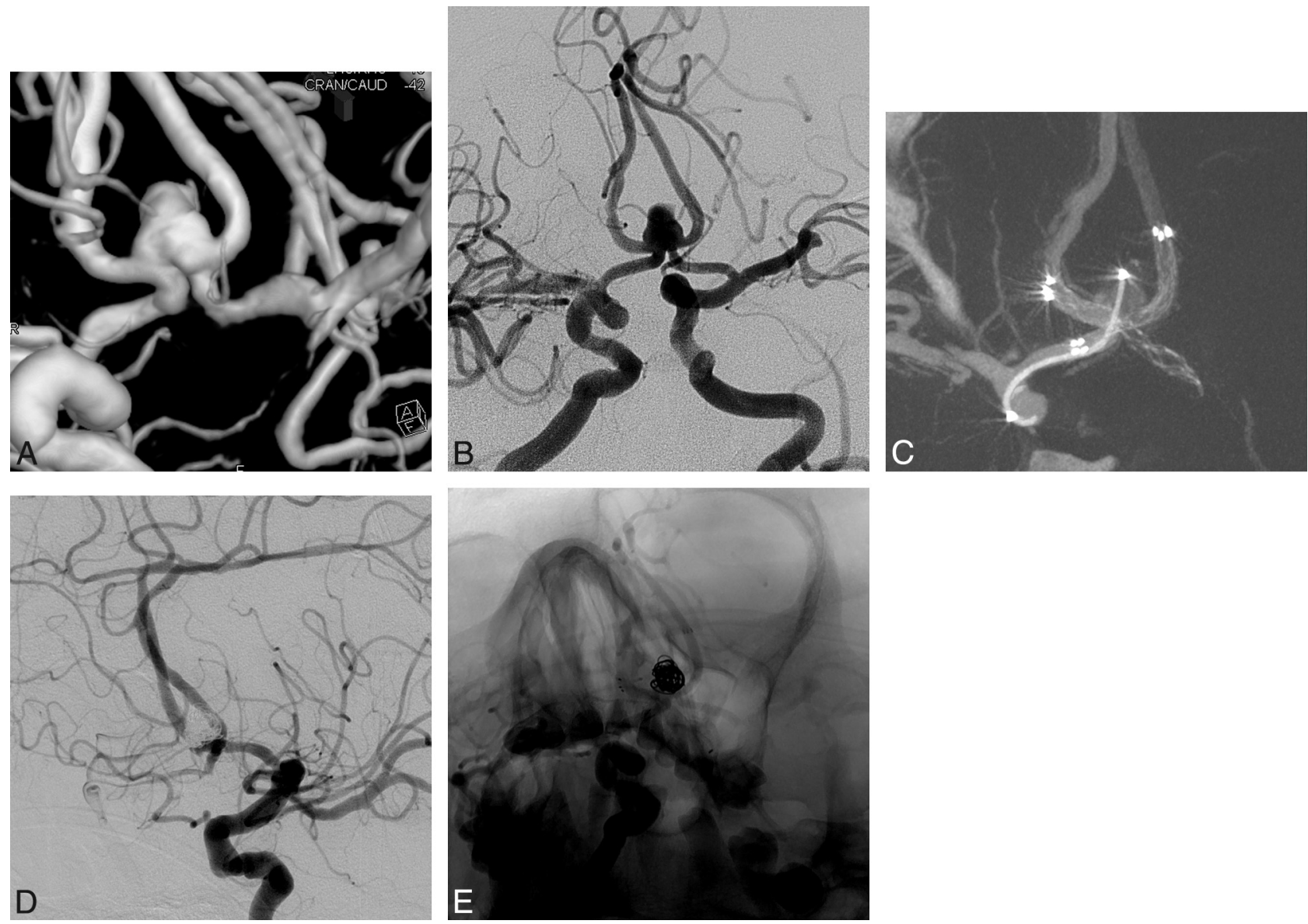

Fig 2. 3D reconstruction $(A)$ and left ICA angiogram $(B)$ show an AcomA aneurysm with a wide neck involving both $A 2$ segments. $C$, DynaCT angiography reveals a satisfactory opening of the Enterprise and Solitaire stents with $X$-configuration. $D$ and $E$, Six-month control angiograms show complete occlusion of the aneurysm.

with excellent reconstruction of the AcomA complex and patency of the stents with no compromise of the parent arteries.

\section{Illustrative Case 2}

A 58-year-old woman was admitted to the hospital with severe headache. Diagnostic work-up, including cerebral angiography with $3 \mathrm{D}$ reconstruction (Fig $2 A,-B$ ), revealed an AcomA aneurysm with a nipple. The aneurysm was $6.5 \times 4 \mathrm{~mm}$ and had a very wide neck of $6 \mathrm{~mm}$ involving both of the A1-A2 junctions. Dual stent support was considered necessary in Xconfiguration for endosaccular coiling. The patient was premedicated with clopidogrel and aspirin with resultant $82 \%$ platelet inhibition. With the patient under general anesthesia, bilateral ICAs were catheterized with $6 \mathrm{~F}$ Envoy guiding catheters. The procedure was performed under roadmap guidance obtained with simultaneous bicarotid contrast injections. A Solitaire stent $(4 \times 20 \mathrm{~mm})$ from the right $\mathrm{A} 2$ to the left $\mathrm{A} 1$ and an Enterprise stent $(4.5 \times 22 \mathrm{~mm})$ from the left A2 to the right A1 were placed, respectively, to create the X-configured stent reconstruction (Fig 2C). The aneurysm was then coiled with bare platinum detachable coils through the microcatheter jailed within the sac before opening of the first stent. The procedure and the postoperative period were uneventful, and the patient was discharged home the next day. Six-month angiography revealed complete obliteration of aneurysm with further thrombosis of the sac, which had had loose packing initially, and the AcomA complex showed excellent reconstruction (Fig 2D, -E) of the stented vessels.

\section{Discussion}

Application of endovascular coil embolization for the treatment of wide-neck cerebral aneurysms has been rapidly evolving subsequent to the introduction of compliant microballoons into clinical use, ${ }^{6}$ thereafter followed by the introduction of neurodedicated self-expanding stents in recent years. Stents are used not only to treat wide-neck aneurysms by the scaffold they provide but also to achieve better initial occlusion rates while sparing the parent artery lumen and to decrease the likelihood of recanalization by the alteration they create in the intra-aneurysmal hemodynamics. ${ }^{7,8}$

The multicenter registry, including preliminary results with the Enterprise stent without follow-up data, revealed a relatively high success rate of stent deployment (failure in 3\%) and $\mathrm{a} \geq 90 \%$ rate of occlusion in $76 \%$ of cases associated with the rates of $2 \%$ mortality and $2.8 \%$ morbidity. ${ }^{4}$ Previously reported smaller series by Weber et $\mathrm{al}^{9}$ and Lubicz et $\mathrm{al}^{10}$ indicated the ease of navigation with the use of the Prowler Plus catheter, excluding the exchange procedure. Precise placement with anchoring flared ends $0.5 \mathrm{~mm}$ larger than the diameter of the stent and the possibility of repositioning are major advantages of the Enterprise stent. Overall, in all 3 series, the Enterprise stent was found to be a safe and an effective 
tool for the treatment of complex intracranial aneurysms, with high initial occlusion rates.

Although the stents for intracranial use were originally designed for mechanical scaffolding purposes, recently appearing laboratory and clinical reports on their hemodynamic effects by alteration of intra-aneurysmal flow velocity are attracting increasing interest. ${ }^{8}$ Laboratory investigation on a patient-specific silicone aneurysm model based on clinical images, according to Tateshima et al, ${ }^{8}$ showed significant alteration of flow direction, velocity, and pattern before and after stent placement. In another in vitro study, the magnitude and pulsatility of the wall shear rate were found to be reduced by stent placement in the lateral aneurysm models. ${ }^{11}$ There have been clinical reports to support these in vitro findings ${ }^{10,12,13}$; Lubicz et $\mathrm{al}^{14}$ reported a $53 \%$ rate of further thrombosis in their series of aneurysms treated with stent-assisted coiling and suggested the adjunctive effect of the stent in stabilizing or significantly improving long-term anatomic results.

During the early experience with the self-expanding intracranial stents, double-stent treatment with a Y-configuration had been introduced to improve the application of coiling to wide-neck basilar bifurcation aneurysms. ${ }^{15}$ Successful initial occlusion rates and follow-up results have been achieved with Y-configured dual-stent-assisted coiling. ${ }^{16}$ With our evolving experience and more meticulous preoperative antiaggregation preparation and assessment, Y-configured dual-stent placement has been part of our routine practice in complex bifurcation aneurysms. Our experience with long-term follow-up of $>70$ patients (unpublished data, S.C., December, 2009) suggested very durable results. We, herein, describe a new dual-stent placement technique of X-configured stent placement for the treatment of AcomA aneurysms with wide-neck complex anatomy located on the AcomA and involving both A1-A2 junctions.

Far beyond the neck size and dome-to-neck ratio, AcomA aneurysms pose different morphologic features for success of initial treatment and long-term durability, such as dome direction, neck location, and the presence of associated anomalies. ${ }^{5}$ Aneurysms with large neck sizes located at the AcomA or with domes directed posteriorly have shown a tendency for greater recanalization rates, independent of the initial occlusion rates. ${ }^{5}$ Birknes et al $^{17}$ analyzed the results of endovascular treatment in their exclusive group of 123 ruptured AcomA aneurysms with respect to aneurysm morphology and reported, overall, a $9.7 \%$ failure of endovascular treatment and a $69.9 \%$ rate of so-called successful embolization ( $\geq 90 \%$ occlusion of the aneurysm sac) in the entire group, $52 \%$ in the group of aneurysms with wider necks ( $\geq 4 \mathrm{~mm}$ ). The recanalization in the follow-up was $33.3 \%$ and $66.7 \%$, respectively, in these patients. To achieve endovascular reconstruction of the complex AcomA aneurysms, adjunctive stent placement has been used to facilitate coil embolization, though it is more limited than that in other aneurysm locations. ${ }^{18}$

AcomA aneurysms are not only challenging for the endovascular techniques but also for microsurgical clipping ${ }^{19}$ and demonstrate the highest incidence of postoperative morbidity among anterior circulation aneurysms. Both A2s emanating from the aneurysm, in addition to the wide base of the aneurysm where the AcomA is partly incorporated within the sac, may pose technical challenges during the microsurgery. In our series, all 5 aneurysms had very wide necks located on the AcomA involving both A1-A2 junctions, and this was the reason that the dual-stent placement was entertained in this group of patients.

Technically, placement of stents from the ipsilateral A2 to the A1 segment might be considered an option; however, we believe that sharp angles between the A1-A2 segments due to very wide-neck and complex anatomy may make application of X-stent placement more feasible in these selected cases. Besides, alteration of flow direction at the neck of the aneurysm, which, hypothetically, has an impact on long-term durability, is thought to be more efficient with the X-configuration rather than ipsilateral placement of stents with "opposite L" configurations. Moreover, the patency of the reconstructed AcomA is preserved, whereas it may become occluded with the latter stent configuration. However, of course, $\mathrm{X}$-stent placement is to be reserved for patients having good-sized A1s, bilaterally. Otherwise, Y-stent placement through the large-sized A1 is the option for patients with hypoplasia/aplasia of A1 on 1 side.

Despite low initial occlusion rates (class $\mathrm{C}$ ) encountered in our patients, follow-up data revealed further thrombosis of all aneurysms into Raymond-Roy class A occlusion. The follow-up results in this small group may indicate not only the favorable mechanical and hemodynamic impact of X-stent placement for AcomA aneurysms but also the roll of the stentcell type in the long-term results. Although, in the series of Thorell et al, ${ }^{16}$ the aneurysms were located at the terminal bifurcations, which were known to be more prone to recanalization, 2 of their 7 aneurysms treated with Y-stent-placement-assisted coiling were recanalized despite initial complete or near-complete occlusion. The open-cell design of the Neuroform stent used in that series may have had an effect on the hemodynamics. We can speculate that the closed-cell design of the stents may result in flow diversion at the intersection point, possibly due to the slight narrowing of the second stent while crossing. On the other hand, we did not encounter any disadvantage of the closed-cell design of the stents used herein, ${ }^{20}$ either during the procedure or in the follow-up results as one may have anticipated, such as constriction of the second stent within the first stent at the intersection point with resultant compromise of the flow or possible narrowing of the lumen at this site in the follow-up. In the literature, in-stent stenosis has been reported in the patients whose aneurysms were treated with stent-assisted coiling. ${ }^{14}$ However, there has been no in-stent stenosis or artery occlusion in this small group of patients, despite dual-stent placement in relatively small vessels.

\section{Conclusions}

This small series with angiographic follow-up results showed that X-configured stent-assisted coiling is an alternative treatment for selected cases.

\section{References}

1. Murayama Y, Nien YL, Duckwiler G, et al. Guglielmi detachable coil embolization of cerebral aneurysms: 11 years' experience. J Neurosurg 2003;98: 959-66

2. Raymond J, Guilbert F, Weill A, et al. Long-term angiographic recurrences after selective endovascular treatment of aneurysms with detachable coils. Stroke 2003;34:1398-403

3. Yavuz K, Geyik S, Pamuk AG, et al. Immediate and midterm follow-up results 
of using an electrodetachable, fully retrievable SOLO stent system in the endovascular coil occlusion of wide-necked cerebral aneurysms. J Neurosurg 2008;109:445-53

4. Mocco J, Synder KV, Albuquerque F, et al. Treatment of intracranial aneurysms with the Enterprise stent: a multicenter registry. J Neurosurg 2009;110: 35-39

5. Gonzalez N, Sedrak M, Martin N, et al. Impact of anatomic features in the endovascular embolization of 181 anterior communicating artery aneurysms. Stroke 2008;39:2776-82

6. Moret J, Cognard C, Weill A, et al. Reconstruction technique in the treatment of wide-neck intracranial aneurysms: long-term angiographic and clinical results-apropos of 56 cases. J Neuroradiol 1997;24:30-44

7. Bendok BR, Parkinson RJ, Hage ZA, et al. The effect of vascular reconstruction device-assisted coiling on packing density, effective neck coverage, and angiographic outcome: an in vitro study. Neurosurgery 2007;61:835-41

8. Tateshima S, Tanishita K, Hakata Y, et al. Alteration of intraaneurysmal hemodynamics by placement of a self-expanding stent. J Neurosurg 2009;111:22-27

9. Weber W, Bendszuz M, Kis B, et al. A new self-expanding nitinol stent (Enterprise) for the treatment of wide-necked intracranial aneurysms: Neuroradiology 2007;49:555-61

10. Lubicz B, François O, Levivier M, et al. Preliminary experience with the Enterprise stent for endovascular treatment of complex intracranial aneurysms: potential advantages and limiting characteristics. Neurosurgery 2008;62:106370, discussion 1069-70

11. Rhee $\mathrm{K}, \mathrm{Han} \mathrm{MH}$, Cha $\mathrm{SH}$. Changes of flow characteristics by stenting in aneurysm models: influence of aneurysm geometry and stent porosity. Ann Biomed Eng 2002;30:894-904
12. Vanninen $\mathrm{R}$, Manninen $\mathrm{H}$, Ronkainen A. Broad-based intracranial aneurysms: thrombosis induced by stent placement. AJNR Am J Neuroradiol 2003;24: 263-66

13. Fiorella D, Albuquerque FC, Deshmukh VR, et al. Endovascular reconstruction with the Neuroform stent as monotherapy for the treatment of uncoilable intradural pseudoaneurysms. Neurosurgery 2006;59:291-300, discussion 291300

14. Lubicz B, Bandeira A, Bruneau M, et al. Stenting is improving and stabilizing anatomical results of coiled intracranial aneurysms. Neuroradiology 2009;51: 419-25

15. Chow MM, Woo HH, Masaryk TJ, et al. A novel endovascular treatment of a wide-necked basilar apex aneurysm by using a Y-configuration, double-stent technique. AJNR Am J Neuroradiol 2004;25:509-12

16. Thorell WE, Chow MM, Woo HH, et al. Y-configured dual intracranial stentassisted coil embolization for the treatment of wide-necked basilar tip aneurysms. Neurosurgery 2005;56:1035-40

17. Birknes JK, Hwang SK, Pandey AS, et al. Feasibility and limitations of endovascular coil embolization of anterior communicating aneurysms: morphological considerations. Neurosurgery 2006;58:43-52

18. Huang Q, Xu Y, Hong B, et al. Stent-assisted embolization of wide-neck anterior communicating aneurysms: review of 21 consecutive cases. AJNR Am J Neuroradiol 2009;30:1502-06

19. Agrawal A, Kato $\mathrm{Y}$, Chen L, et al. Anterior communicating artery aneurysms: an overview. Minim Invasive Neurosurg 2008;51:131-35

20. Ebrahimi N, Claus B, Lee CY, et al. Stent conformity in curved vascular models with simulated aneurysm necks using flat-panel $\mathrm{CT}$ : an in vitro study. AJNR Am I Neuroradiol 2007;28:823-29 